\title{
Improved Noninterferometric Test of Collapse Models Using Ultracold Cantilevers
}

\author{
A. Vinante, ${ }^{1, *}$ R. Mezzena, ${ }^{2,5}$ P. Falferi, ${ }^{1,5}$ M. Carlesso, ${ }^{3,4}$ and A. Bassi ${ }^{3,4}$ \\ ${ }^{1}$ Istituto di Fotonica e Nanotecnologie, CNR-Fondazione Bruno Kessler, I-38123 Povo, Trento, Italy \\ ${ }^{2}$ Department of Physics, University of Trento, I-38123 Povo, Trento, Italy \\ ${ }^{3}$ Department of Physics, University of Trieste, Strada Costiera 11, 34151 Trieste, Italy \\ ${ }^{4}$ Istituto Nazionale di Fisica Nucleare, Trieste Section, Via Valerio 2, 34127 Trieste, Italy \\ ${ }^{5}$ Istituto Nazionale di Fisica Nucleare, TIFPA, I-38123 Povo, Trento, Italy
}

(Received 21 March 2017; published 12 September 2017)

\begin{abstract}
Spontaneous collapse models predict that a weak force noise acts on any mechanical system, as a consequence of the collapse of the wave function. Significant upper limits on the collapse rate have been recently inferred from precision mechanical experiments, such as ultracold cantilevers and the space mission LISA Pathfinder. Here, we report new results from an experiment based on a high- $Q$ cantilever cooled to millikelvin temperatures, which is potentially able to improve the current bounds on the continuous spontaneous localization (CSL) model by 1 order of magnitude. High accuracy measurements of the cantilever thermal fluctuations reveal a nonthermal force noise of unknown origin. This excess noise is compatible with the CSL heating predicted by Adler. Several physical mechanisms able to explain the observed noise have been ruled out.
\end{abstract}

DOI: 10.1103/PhysRevLett.119.110401

Spontaneous wave function collapse models [1-4] are stochastic nonlinear modifications of standard quantum mechanics, which have been introduced as a possible solution of the measurement problem. According to such models, the stochastic collapse of the wave function is a dynamical process which naturally breaks the quantum superposition principle. The process would occur in atomic systems on a very long time scale, practically unobservable, so that standard quantum mechanics would hold strictly. However, the scaling of the collapse rate with the size of the system would lead to a rapid localization of any macroscopic system, and to the emergence of the definiteness of the classical everyday world.

Here we consider the continuous spontaneous localization (CSL) model [2]. CSL is the most known and studied collapse model and has been extensively reviewed in many recent papers [3,4]. It is characterized by two phenomenological constants, a collapse rate $\lambda$ and a length $r_{C}$, which characterize, respectively, the intensity and the spatial resolution of the spontaneous collapse. The conservative value for the collapse rate suggested by Ghirardi, Rimini, and Weber $[1,2]$ is $\lambda \simeq 10^{-16} \mathrm{~s}^{-1}$ at $r_{C}=10^{-7} \mathrm{~m}$, and is obtained by imposing the collapse to be effective at the macroscopic human scale. A collapse rate $10^{9 \pm 2}$ times larger has been suggested by Adler [5], motivated by the requirement of making the wave-function collapse effective at the mesoscopic level.

Direct laboratory tests based on quantum superposition experiments set limits on $\lambda$ at the level of $10^{-6} \mathrm{~s}^{-1}$ [6-8]. Much stronger bounds can be set by indirect noninterferometric tests based on mechanical systems [9-16]. Relevant mechanical bounds on $\lambda$ at the level of $10^{-8} \mathrm{~s}^{-1}$ for
$r_{C}=10^{-7} \mathrm{~m}$ have been recently set by cantilever-based experiments [17], cold atoms [18,19], and the space-based experiment LISA Pathfinder [20-22]. Stronger bounds, though less robust to variations on the model $[23,24]$, are set by spontaneous emission of $\mathrm{x}$ rays [25].

Here, we report on a improved version of the cantilever experiment, with a relative strength of the thermal noise force reduced by 1 order of magnitude. Unlike the previous cantilever experiment [17], we find evidence of a nonthermal excess noise of unknown origin. If interpreted as CSL-induced noise, this would be compatible with previous experimental bounds and in agreement with the collapse rate predicted by Adler [5]. Alternatively, if the noise finds an explanation within standard physics, its identification and elimination will allow us to extend the experimental bound on $\lambda$ by 1 order of magnitude, almost ruling out Adler's proposal.

The scheme of our experiment is similar to the one described in Ref. [17] and is shown in Fig. 1. A cantilever with a spherical ferromagnetic load is continuously monitored by a superconducting quantum interference device (SQUID). The displacement $x$ is converted into magnetic flux by a linear coupling $\Phi_{x}=d \Phi / d x$ which depends on the magnet position and orientation. A novel feature is that the mechanical quality factor $Q$ is much higher than in previous experiments, and heavily temperature dependent. Moreover, we observe a dynamical SQUID-induced magnetic spring effect, analog to optical spring effects in optomechanics, which modifies the quality factor from its intrinsic value $Q$ to an apparent value $Q_{a}$ [26]. To account for this new feature, we seek a strategy to directly measure the effective force noise 


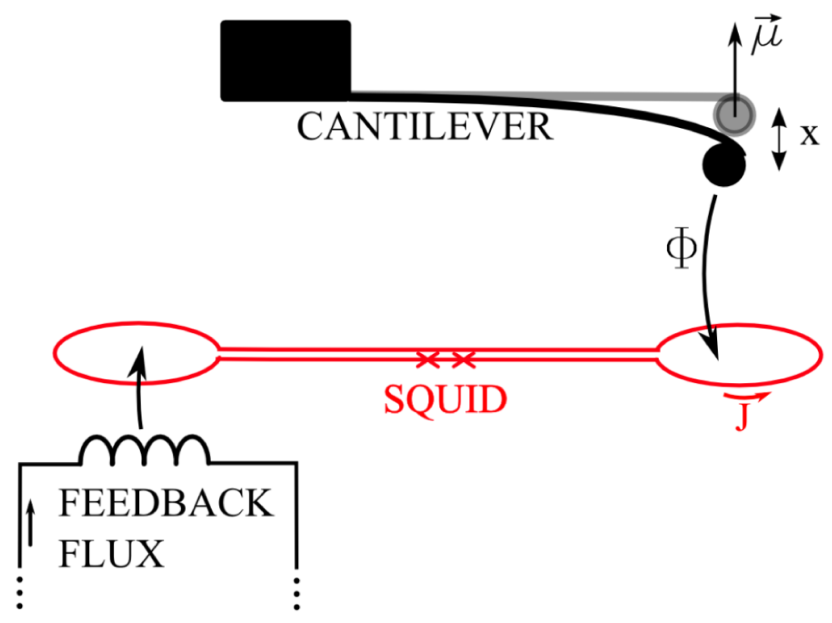

FIG. 1. Simplified measurement scheme. The fundamental bending mode of a cantilever loaded with a ferromagnetic microsphere with magnetic moment $\mu$ is continuously monitored by a SQUID susceptometer. The SQUID measures the magnetic flux $\Phi=\Phi_{x} x$ coupled by the displacement $x$ of the magnetic particle and is operated in a flux-locked loop (feedback electronics are not shown, for simplicity). The flux-dependent circulating current $J$, combined with finite feedback gain, causes a dynamical magnetic spring effect that modifies the apparent quality factor of the cantilever.

acting on the resonator, rather than the mean energy which may be affected by the dynamically modified quality factor $Q_{a}$. To this end, let us consider the Lorentzian spectral density associated with the cantilever displacement fluctuations,

$$
S_{x}=\left(\frac{S_{F 0}}{k^{2}}+\frac{4 k_{B} T}{k \omega_{0} Q}\right) \frac{f_{0}^{4}}{\left(f_{0}^{2}-f^{2}\right)^{2}+\left(f f_{0} / Q_{a}\right)^{2}} .
$$

Here, $f_{0}=\omega_{0} / 2 \pi$ is the resonant frequency, $k$ is the spring constant, $k_{B}$ is the Boltzmann constant, $T$ is the temperature, and $S_{F 0}$ is the spectral density of any nonthermal force noise. Equation (1) says that magnetic spring effects, similar to optomechanical ones, only affect the dynamics and thus the denominator of the resonant term, characterized by an apparent quality $Q_{a}$ [27-29]. Instead, the amplitude of the Lorentzian curve is proportional to the total force noise. The thermal contribution scales with $T / Q$, where $Q$ is the intrinsic quality factor. Our strategy is to characterize the thermal noise by accurate measurements as function of $T / Q$. Any excess nonthermal noise, included that induced by CSL, will cause a constant force spectral density $S_{F 0}$, independent of $T / Q$. The maximum nonthermal force noise compatible with the experiment can be used to test CSL predictions. This requires modeling the CSL force acting on the continuous mechanical resonator, exactly as done in Ref. [17] (see Supplemental Material for details [30]).
The mechanical resonator in our setup is a commercial tipless atomic force microscopy silicon cantilever, with size $450 \times 57 \times 2.5 \mu \mathrm{m}^{3}$ and stiffness $k=(0.40 \pm 0.02) \mathrm{N} / \mathrm{m}$. A hard ferromagnetic microsphere (radius $R=15.5 \mu \mathrm{m}$, density $\rho=7.43 \mathrm{~kg} / \mathrm{m}^{3}$ ) is glued to the cantilever free end and magnetized. The microsphere has a twofold function. It increases the cross section to the CSL field, which scales as $\rho^{2}$ [17], while at the same time enabling a straightforward detection by means of a nearby SQUID susceptometer. The SQUID is gradiometric and comprises two distant loops with radius $R_{S}=10 \mu \mathrm{m}$ [32]. The particle is aligned above the first loop at a height $h \simeq 40 \mu \mathrm{m}$, with the motion of the first flexural mode orthogonal to the SQUID plane. The SQUID is operated in two-stage flux-locked-loop configuration with the feedback applied to the second loop (see Fig. 1). This geometry strongly suppresses direct coupling between the feedback signal and the cantilever.

The cantilever-SQUID system is enclosed in a copper box, suspended above the mixing chamber plate of a pulsetube dilution refrigerator (Janis Jdry-100-Astra) by means of a two-stage suspension system. The measured mechanical attenuation is higher than $80 \mathrm{~dB}$ at the cantilever frequency. The temperature of the mixing chamber is measured by $\mathrm{RuO}_{2}$ thermometer, while the temperature of the SQUID box is measured by a SQUID noise thermometer. Both devices have been calibrated against a superconducting reference point device with accuracy better than $0.5 \%$.

The resonant frequency of the fundamental mode of the cantilever is $f_{0}=8174.01 \mathrm{~Hz}$. The actual mechanical quality factor $Q_{a}$ is determined by ringdown measurements. The dynamical magnetic spring effect depends on the SQUID working point and scales as $1 /|G|$ where $G$ $(|G| \gg 1)$ is the open loop gain of the feedback electronics. Specifically, we expect a linear dependence of the actual quality factor $Q_{a}$ as $1 / Q_{a}=1 / Q+c /|G|$, where $c$ is a constant depending on the SQUID working point. This behavior is experimentally observed by varying the gain $|G|$, and allows us to infer the intrinsic quality factor $Q$. The whole measurement procedure is repeated at each temperature $T$ [30].

The measured intrinsic $Q$ is of the order of $6 \times 10^{5}$ at temperature of the order of $1 \mathrm{~K}$, and is observed to increase roughly as $1 / T$ upon reducing the temperature below $T=500 \mathrm{mK}$, approaching $Q \simeq 10^{7}$ at $T \simeq 20 \mathrm{mK}$. This behavior is consistent with measurements performed with the SQUID weakly coupled, and is reminiscent of two-level systems dissipation [33]. Standard glassy two-level systems in a 2-nm amorphous oxide layer on the cantilever surface are able to explain the observed effect quantitatively.

The noise is measured by acquiring and averaging spectra of the SQUID signal, calibrated as magnetic flux, with typical integration time of 800-1200 s. Before measuring the noise and the quality factor, the system is allowed to thermalize for at least 3 hours. During the noise measurement the pulse 


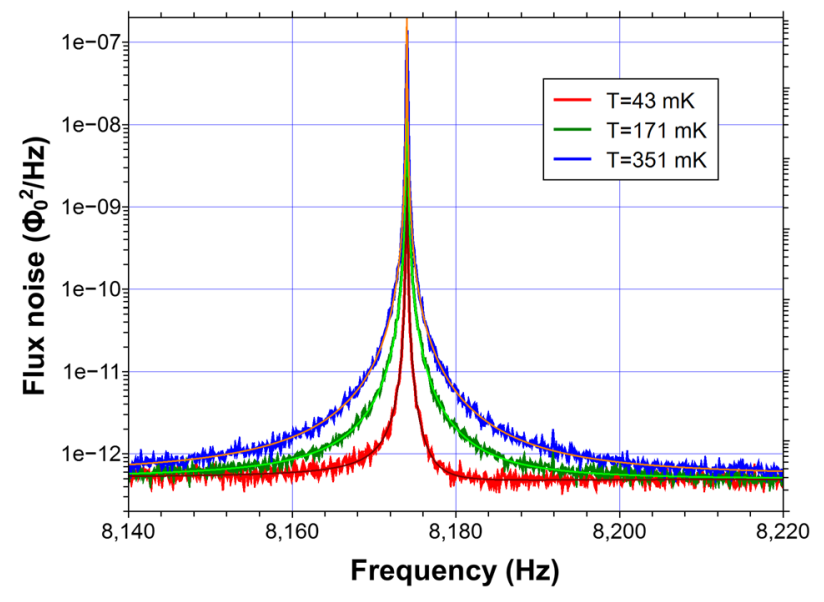

FIG. 2. Examples of averaged spectra at three representative temperatures, with the respective best fit with Eq. (2).

tube is switched off, and the mixing chamber temperature actively stabilized by a proportional-integral-derivative controller. Examples of averaged flux noise spectra at three representative temperatures are shown in Fig. 2. The spectra are fitted with the curve

$$
S_{\Phi}=A+\frac{B f_{0}^{4}+C\left(f^{2}-f_{1}^{2}\right)^{2}}{\left(f^{2}-f_{0}^{2}\right)^{2}+\left(f f_{0} / Q_{a}\right)^{2}} .
$$

The term proportional to $B$ is the relevant one, as it corresponds to the fluctuations of the cantilever induced by thermal or extra force noise, given by Eq. (1), converted into magnetic flux. The term proportional to $A$ is the purely additive wideband noise of the SQUID. The last term, proportional to $C$, arises because of the flux noise applied to the SQUID by the feedback electronics in order to compensate for the SQUID additive wideband noise. This flux noise induces a current $J$ circulating in the SQUID through the finite responsivity $J_{\Phi}=d J / d \Phi$ [26], which eventually leads to an effective backaction on the cantilever. The transfer function of this mechanism has been directly measured by injecting a calibration signal and features an antiresonance at $f=f_{1}$, with $f_{1}-f_{0}=1.1 \mathrm{~Hz}$. The overall effect is a small asymmetric distortion of the Lorentzian peak.

All spectra have been checked by $\chi^{2}$ tests [30] to be consistent with Eq. (2). All estimations of the SQUID parameters $A$ and $C$ are consistent with each other, with mean values $A=(1.23 \pm 0.05) \times 10^{-13} \Phi_{0}^{2} / \mathrm{Hz}$ and $C=(3.78 \pm 0.05) \times 10^{-13} \Phi_{0}^{2} / \mathrm{Hz}$. In particular, $A$ and $C$ do not depend significantly on temperature. This is expected, as for this type of SQUID [29] the noise is saturated by hot electron effect [34] for $T<400 \mathrm{mK}$, and our measurements satisfy this condition.

Figure 3 shows the measured symmetric amplitude $B$ of the Lorentzian noise as function of $T / Q$, varied by changing the bath temperature. The uncertainty on the estimation of $B$ is remarkably low, of the order of $1 \%$. The $x$-error bar, dominated by the uncertainty on $Q$, is thus

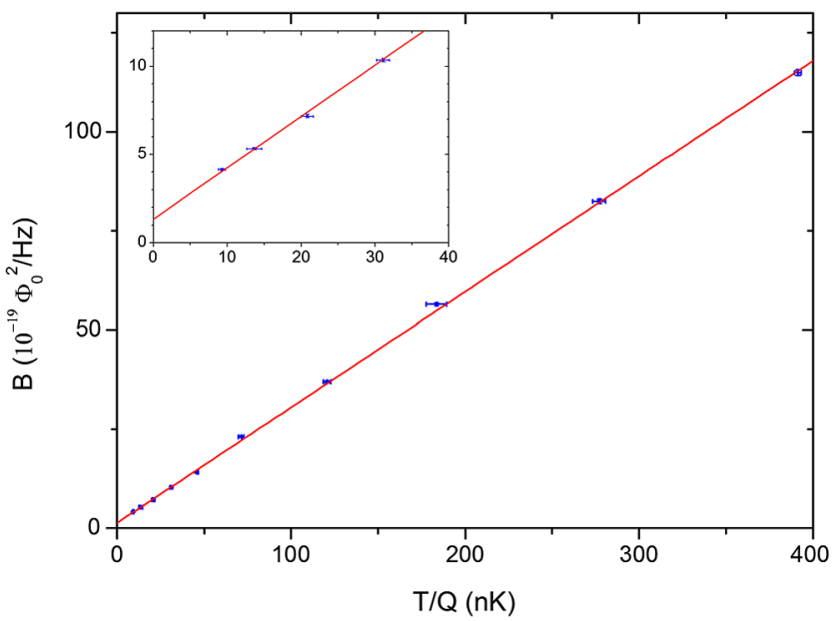

FIG. 3. Symmetric amplitude of the Lorentzian noise $B$, as measured by the SQUID, as function of the ratio $T / Q$, together with the best linear fit. In the inset, the data at the lowest $T / Q$ are zoomed in order to highlight the nonzero intercept of the fit.

significant [30]. The data agree with a linear behavior over the whole $T / Q$ range, in agreement with Eq. (1). A weighted orthogonal linear fit with the expression $B_{0}+B_{1} T / Q$ yields the intercept $B_{0}=(1.27 \pm 0.11) \times$ $10^{-19} \Phi_{0}^{2} / \mathrm{Hz}$ and the slope $B_{1}=(0.291 \pm 0.002) \times$ $10^{-19} \Phi_{0}^{2} /(\mathrm{nKHz})$. In addition, we exploit the linear dependence on $T / Q$ to infer the coupling between cantilever and SQUID. Given Eqs. (1) and (2) we can express the thermal slope $B_{1}$ as

$$
B_{1}=\frac{4 k_{B}}{\omega_{0}} \frac{\Phi_{x}^{2}}{k}
$$

which allows the coupling factor $\Phi_{x}^{2} / k$ to be evaluated from the measured $B_{1}$.

The finite intercept, clearly visible in the inset of Fig. 3, implies that the data are not compatible with a pure thermal noise behavior, and a nonthermal excess noise is present. According to Eq. (1) we can convert $B_{0}$ into a residual force noise,

$$
S_{F 0}=\frac{4 k_{B} k}{\omega_{0}} \frac{B_{0}}{B_{1}} .
$$

The measured coupling factor and residual force noise are reported in Table I, together with the same quantities inferred from the additional measurements discussed in the following. The systematic error on $S_{F 0}$ arises from the uncertainty on $k$.

We checked for possible physical sources of the excess force noise. First, we expect a backaction force spectral density $S_{F, B A}=S_{J} F_{J}^{2}$ from the noise in the current circulating in the SQUID loop. Here, $S_{J}$ is the current spectral density and $F_{J}=d F / d J$ is the backward current-to-force factor. Because of reciprocity, $F_{J}$ must be equal to the 
TABLE I. Operating conditions, measured coupling, and residual force noise for the different measurement data sets. Sys represents the estimated systematic error.

\begin{tabular}{lcc}
\hline \hline Pulse tube & $\Phi_{x}^{2} / k(\mathrm{fH})$ & $S_{F 0}\left(\mathrm{aN}^{2} / \mathrm{Hz}\right)$ \\
\hline Off & $116 \pm 1$ & $1.87 \pm 0.16 \pm 0.1(\mathrm{sys})$ \\
Off & $347 \pm 3$ & $2.12 \pm 0.20 \pm 0.1(\mathrm{sys})$ \\
On & $114 \pm 2$ & $2.58 \pm 0.20 \pm 0.1(\mathrm{sys})$ \\
\hline \hline
\end{tabular}

forward displacement-to-flux factor $\Phi_{x}$ [35] so that $S_{F, B A}=S_{J} \Phi_{x}^{2}$. In other words, the backaction noise leads to a finite intercept, and the corresponding force noise scales with the coupling factor.

We took advantage of this property and performed additional measurements in a subsequent cooldown at a different cantilever position, with effective coupling increased by a factor of $\sim 3$. We observe again a linear behavior in very good agreement with the experimental data, with a finite intercept [30]. The corresponding residual force noise is reported in the second row of Table I and is consistent within the error bar with the one at low coupling. This clearly indicates that most of the observed excess noise cannot be attributed to SQUID backaction.

We can compare this result with the prediction of the Clarke-Tesche model [36] for the current noise, $S_{J}=$ $\gamma k_{B} T_{\mathrm{SQ}} / R_{\mathrm{SQ}}$ with $\gamma \simeq 11$ for an optimized SQUID. Here $R_{\mathrm{SQ}}=8 \Omega$ and $T_{\mathrm{SQ}} \simeq 400 \mathrm{mK}$ are the measured shunt resistor and the typical SQUID electron temperature. From this expression we estimate a small increase of the backaction force noise $\Delta S_{F, B A} \simeq 0.6 \mathrm{aN}^{2} / \mathrm{Hz}$ between the two measurements, which is compatible within $2 \sigma$ with the experimental increase $\Delta S_{F 0}=(0.24 \pm 0.26) \mathrm{aN}^{2} / \mathrm{Hz}$.

In order to investigate the role of vibrational noise from the refrigerator or from the outside world, we repeated the measurements at low coupling by keeping the pulse tube on [30]. The input mechanical noise provided by the pulse tube in our cryostat is known to be 2-3 orders of magnitude larger than the background noise when the pulse tube is off. However, while the measured spectra with the pulse tube on are significantly dirtier, we can still perform a Lorentzian fit and the residual force noise, reported in Table I, is only slightly increased with respect to the measurements with the pulse tube off. This confirms that the mechanical suspensions are working well within design specifications, and suggests that vibrational noise is not the source of the observed excess noise with the pulse tube off. We have also ruled out vibrational noise from the ${ }^{3} \mathrm{He}$ flow, by switching the circulation pump on and off without noticeable effects [30].

Magnetic effects, such as fluctuations of the environmental magnetic field or fluctuations of the microsphere magnetization, can be also considered as possible excess noise sources. We can substantially rule out these mechanisms, based on theoretical order-of-magnitude estimations, and a further test which has shown the quality factor to be independent of the external static field [30].

Another option is that we are actually observing thermomechanical noise, but the effective temperature of the noise source (or part of it) is higher than the one of the thermal bath because of thermal gradients along the cantilever. In this case one would expect to observe saturation effects, as observed in [17] rather than a linear behavior with a fixed intercept. Furthermore, we have performed simple thermal modeling of the cantilever. The power dissipated in the magnet by eddy currents induced by SQUID Josephson radiation is estimated to be of the order of $1 \mathrm{fW}$, and would cause a temperature gradient between the magnet and the cantilever base smaller than $1 \mathrm{mK}$ in the temperature range explored by this experiment.

The observed finite intercept could also be a subtle artifact due to an unknown systematic error in the determination of $1 / Q$. We find that this is in principle possible, but the systematic error on $1 / Q$ would have to be 10 times larger than the statistical error bar to be consistent with zero excess noise. Moreover, the data would no longer follow a linear behavior [30].

Finally, let us compare our results with the predictions of the CSL model. By using the same method discussed in Ref. [17], we can convert the observed excess noise into the red curve in the parameter space $\lambda-r_{C}$ of the CSL model, shown in Fig. 4. This curve can be considered

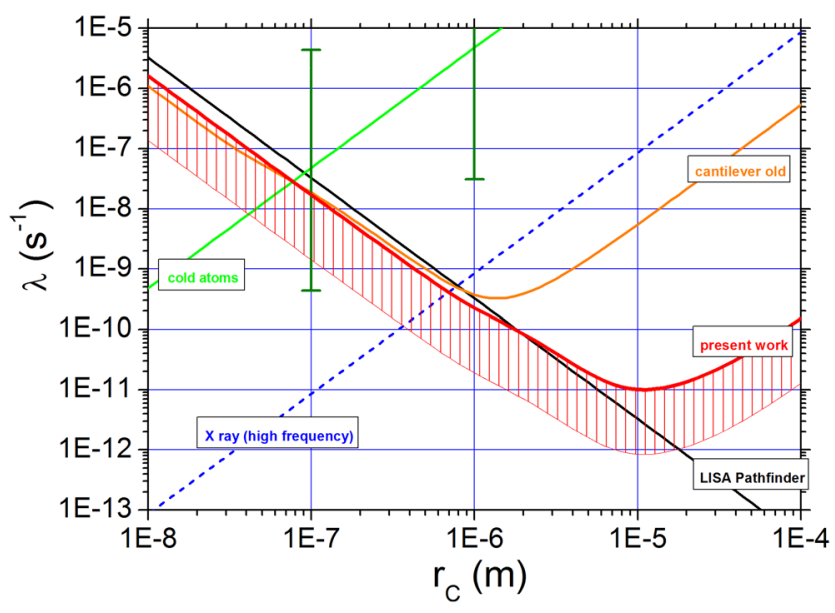

FIG. 4. Exclusion plot in the $\lambda-r_{C}$ plane based on our experimental data, compared with the best experimental upper bounds reported so far and with theoretical predictions. Continuous thick (red) curve: CSL collapse rate $\lambda$, as function of the characteristic length $r_{C}$, assuming that the observed noise is entirely due to CSL. The shaded region would be excluded by our experiment if the physical origin of the excess noise were identified. The other thin lines represent upper limits from labeled experiments: previous cantilever experiment (orange) [17], LISA Pathfinder (black) [21], cold atoms (green) [19], and x-ray spontaneous emission (dashed blue) [25]. The (dark green) bars represent the CSL collapse rate suggested by Adler [5]. 
as a conservative improved upper bound from mechanical experiments for $r_{C} \in\left[10^{-7}, 2 \times 10^{-6}\right] \mathrm{m}$. If the excess noise were indeed due to CSL, the true CSL parameters would actually lie somewhere on the curve. For the standard choice $r_{C}=10^{-7} \mathrm{~m}$ this would imply $\lambda=10^{-7.7} \mathrm{~s}^{-1}$, in agreement with Adler's predictions [5]. Alternatively, if the observed noise can be eventually reduced to standard physical effects, its identification and elimination will lead to an improved upper bound on CSL, determined by the experimental error bar. The parameter region which can be potentially excluded is shaded in Fig. 4. A full exclusion would almost completely rule out Adler's predictions [5].

In conclusion, we have performed an improved cantilever-based test of the CSL model. The new experiment features excess noise, and is in principle compatible with the predictions by Adler [5]. Several physical mechanisms able to explain the observed excess noise have been ruled out. Further investigations are needed in order to probe other possible explanations. Besides further analysis of the present experiment, it will be important to repeat the experiment with a modified setup, for instance, with a different cantilever or microsphere, and other groups should possibly repeat similar measurements with a different setup. Above all, this experiment neatly illustrates the fundamental challenge of collapse model testing. Negative results are robust, but positive claims require extremely careful and systematic work in order to exclude any conceivable alternative physical explanation.

A. V. thanks the organizers of the Fundamental Problems in Quantum Physics Program (Bangalore, India) for support, and thanks many participants for useful discussions, in particular, Hendrik Ulbricht, Tejinder Singh, Tjerk Oosterkamp, Nikolai Kiesel, Saikat Ghosh, and Daniel Sudarsky. A. B. and M. C. acknowledge financial support from the University of Trieste (FRA 2016) and INFN.

*andrea.mistervin@gmail.com

[1] G. C. Ghirardi, A. Rimini, and T. Weber, Phys. Rev. D 34, 470 (1986).

[2] G. C. Ghirardi, P. Pearle, and A. Rimini, Phys. Rev. A 42, 78 (1990); G. C. Ghirardi, R. Grassi, and F. Benatti, Found. Phys. 25, 5 (1995).

[3] A. Bassi and G. C. Ghirardi, Phys. Rep. 379, 257 (2003).

[4] A. Bassi, K. Lochan, S. Satin, T. P. Singh, and H. Ulbricht, Rev. Mod. Phys. 85, 471 (2013).

[5] S. L. Adler, J. Phys. A 40, 2935 (2007).

[6] K. Hornberger, S. Gerlich, P. Haslinger, S. Nimmrichter, and M. Arndt, Rev. Mod. Phys. 84, 157 (2012).

[7] T. Juffmann, H. Ulbricht, and M. Arndt, Rep. Prog. Phys. 76, 086402 (2013).

[8] M. Arndt and K. Hornberger, Nat. Phys. 10, 271 (2014).
[9] B. Collett and P. Pearle, Found. Phys. 33, 1495 (2003).

[10] S. L. Adler, J. Phys. A 38, 2729 (2005).

[11] A. Bassi, E. Ippoliti, and S. L. Adler, Phys. Rev. Lett. 94, 030401 (2005).

[12] M. Bahrami, M. Paternostro, A. Bassi, and H. Ulbricht, Phys. Rev. Lett. 112, 210404 (2014).

[13] S. Nimmrichter, K. Hornberger, and K. Hammerer, Phys. Rev. Lett. 113, 020405 (2014).

[14] L. Diosi, Phys. Rev. Lett. 114, 050403 (2015).

[15] D. Goldwater, M. Paternostro, and P. F. Barker, Phys. Rev. A 94, 010104 (2016).

[16] J. Li, S. Zippilli, J. Zhang, and D. Vitali, Phys. Rev. A 93, 050102 (2016).

[17] A. Vinante, M. Bahrami, A. Bassi, O. Usenko, G. Wijts, and T.H. Oosterkamp, Phys. Rev. Lett. 116, 090402 (2016).

[18] T. Kovachy, J. M. Hogan, A. Sugarbaker, S. M. Dickerson, C. A. Donnelly, C. Overstreet, and M. A. Kasevich, Phys. Rev. Lett. 114, 143004 (2015).

[19] M. Bilardello, S. Donadi, A. Vinante, and A. Bassi, Physica A (Amsterdam) 462A, 764 (2016).

[20] M. Armano et al., Phys. Rev. Lett. 116, 231101 (2016).

[21] M. Carlesso, A. Bassi, P. Falferi, and A. Vinante, Phys. Rev. D 94, 124036 (2016).

[22] B. Helou, B. Slagmolen, D. E. McClelland, and Y. Chen, Phys. Rev. D 95, 084054 (2017).

[23] S. L. Adler and F. Ramanazoglu, J. Phys. A 40, 13395 (2007).

[24] S. Donadi and A. Bassi, J. Phys. A 48, 035305 (2015).

[25] C. Curceanu, B. C. Hiesmayr, and K. Piscicchia, Adv. Phys. 4, 263 (2015).

[26] C. Hilbert and J. Clarke, J. Low Temp. Phys. 61, 237 (1985).

[27] M. Poot and H. S. J. Van der Zant, Phys. Rep. 511, 273 (2012).

[28] M. Aspelmeyer, T. J. Kippenberg, and F. Marquardt, Rev. Mod. Phys. 86, 1391 (2014).

[29] P. Falferi, M. Bonaldi, M. Cerdonio, R. Mezzena, G. A. Prodi, A. Vinante, and S. Vitale, Appl. Phys. Lett. 93, 172506 (2008).

[30] See Supplemental Material at http://link.aps.org/ supplemental/10.1103/PhysRevLett.119.110401 for details on CSL noise calculation, data analysis and additional measurements, which includes Ref. [31].

[31] B. C. Stipe, H. J. Mamin, C. S. Yannoni, T. D. Stowe, T. W. Kenny, and D. Rugar, Phys. Rev. Lett. 87, 277602 (2001).

[32] A. Vinante and P. Falferi, Phys. Rev. Lett. 111, 207203 (2013).

[33] A. D. Fefferman, R. O. Pohl, A. T. Zehnder, and J. M. Parpia, Phys. Rev. Lett. 100, 195501 (2008).

[34] F. C. Wellstood, C. Urbina, and J. Clarke, Phys. Rev. B 49, 5942 (1994).

[35] O. Usenko, A. Vinante, G. Wijts, and T. H. Oosterkamp, Appl. Phys. Lett. 98, 133105 (2011).

[36] C. D. Tesche and J. Clarke, J. Low Temp. Phys. 37, 397 (1979). 